\title{
Energy Saving by Blockchaining Maintenance
}

\author{
Michele Albano ${ }^{1, *}$, Pankaj Sharma ${ }^{2}$, Jaime Campos ${ }^{3}$ \\ and Erkki Jantunen ${ }^{4}$
}

${ }^{1}$ CISTER, ISEP, Polytechnic Institute of Porto, Portugal

${ }^{2}$ Department of Industrial Systems Engineering and Management, Faculty of Engineering, National University of Singapore, Singapore

${ }^{3}$ Department of Informatics, Linnaeus University, Sweden

${ }^{4}$ VTT Technical Research Centre of Finland Ltd, Finland

E-mail:mialb@isep.ipp.pt; pankajsharma@nus.edu.sg; jaime.campos@lnu.se; erkki.jantunen@vtt.fi

${ }^{*}$ Corresponding Author

Received 27 September 2018; Accepted 04 October 2018; Publication 08 January 2019

\begin{abstract}
The development and interest in Industry 4.0 together with rapid development of Cyber Physical Systems has created magnificent opportunities to develop maintenance to a totally new level. The Maintenance 4.0 vision considers massive exploitation of information regarding factories and machines to improve maintenance efficiency and efficacy, for example by facilitating logistics of spare parts, but on the other hand this creates other logistics issues on the data itself, which only exacerbate data management issues that emerge when distributed maintenance platforms scale up. In fact, factories can be delocalized with respect to the data centers, where data has to be transferred to be processed. Moreover, any transaction needs communication, be it related to purchase of spare parts, sales contract, and decisions making in general, and it has to be verified by remote parties. Keeping in mind the current average level of Overall Equipment Efficiency (50\%) i.e. there is a hidden factory behind every factory, the potential is huge. It is expected that most of this potential can be realised based on the use
\end{abstract}

Journal of Industrial Engineering and Management Science, Vol. 1, 63-88. doi: 10.13052/jiems2446-1822.2018.004

This is an Open Access publication. (c) 2019 the Author(s). All rights reserved. 
of the above named technologies, and relying on a new approach called blockchain technology, the latter aimed at facilitating data and transactions management. Blockchain supports logistics by a distributed ledger to record transactions in a verifiable and permanent way, thus removing the need for multiple remote parties to verify and store every transaction made, in agreement with the first " $r$ " of maintenance (reduce, repair, reuse, recycle). Keeping in mind the total industrial influence on the consumption of natural resources, such as energy, the new technology advancements can allow for dramatic savings, and can deliver important contributions to the green economy that Europe aims for. The paper introduces the novel technologies that can support sustainability of manufacturing and industry at large, and proposes an architecture to bind together said technologies to realise the vision of Maintenance 4.0.

Keywords: OEE, Blockchain, CPS, IoT, Maintenance.

\section{Introduction}

Overall equipment effectiveness (OEE) is a popular metric that is used for evaluating the equipment effectiveness in a manufacturing environment. The measure was originally presented and explained by (Nakajima, 1988). He defined OEE as the measure for "unlocking the hidden factory" and improving resource utilization. (Jeong and Phillips, 2001) view OEE as a powerful benchmarking key performance indicator (KPI) focusing on three efficiencies; availability, performance and quality. Nakajima's original work identified six losses that reduced the utilization of a machine for the purpose of manufacturing. These losses are equipment failures, setup and adjustments, idling and minor stoppages, reduced speed, defects in process, and reduced yield (Nakajima, 1988). There have been certain alterations/additions to the original definition by Nakajima. Works like (Blanchard, 1997) and (Ingemansson, 2004) added stoppages like preventive maintenance and shortage of staff in order to calculate a more accurate OEE. (Robinson and Ginder, 1995) suggested seven stoppages by replacing "defects in process" stoppage of Nakajima with two different stoppages, namely time lost to inefficient start-up and time lost to tooling. (The and Johnston, 2015) combined some of these factors and included a few others to arrive at four operational production losses. These are Loss due to lack of demand for products, Loss due to availability of equipment, Loss due to slow or sub-optimal performance 
of process or equipment and Loss due to production of poor quality or recovery of product. Irrespective of the number and types of these losses, they are formulated as a function of a number of mutually exclusive components namely: availability (A), performance (P) and quality (Q) (Garza-Reyes, 2015). OEE is the result achieved by multiplying these three factors together:

$$
\mathrm{OEE}=[\text { Availability } * \text { Performance } * \text { Quality }]
$$

Academia has differing views on what this OEE should be and what it practically is. (Nakajima, 1988) indicated that a good benchmark for manufacturing organizations is to have an OEE of 0.84. (Kotze, 1993) puts a figure of OEE less than 0.50 as being closer to the reality. (Ericsson, 1997) found out that OEE can vary in different firms from 0.30 to 0.80 . (Blanchard, 1997) puts a figure of 0.85 as the world class OEE whereas (Ingemansson, 2004) reports the average OEE to be around 50 per cent. (Ylipaa et al., 2017) analyzed 94 empirical data sets from the manufacturing industry between 2006 and 2012 and found the average OEE to be 51.5 per cent. (Parida et al., 2014) argue that the OEE is generally 15-25 per cent below the targeted level. (Edward and Hartmann, 1992) propose that within most plants there is a hidden factory offering some 25-30 per cent more capacity. OEE is the measure that allows a calculation to be made of the current equipment efficiency and more importantly the improvement potential within the equipment.

New technologies that are changing the game for industries have the capability to do the same to the improvement in OEE and productivity. Cyber Physical Systems have made the computation of the physical quantities a reality. Both blockchain and Internet of Things (IoT) have the potential to further aid in the implementation of sound maintenance practices. This paper is the continuation of a work (Albano, Jantunen et al., 2018) that discussed, for the first time to our knowledge, the application of these technologies to maintenance, and it extends the previous work with some insights regarding the efficiency that can be introduced with respect to traditional approaches. This paper will explain these newer technologies and will illustrate how they can be used in asset management field. This section briefly discusses about the sustainability of the manufacturing field followed by how a better OEE can lead to more sustainable manufacturing. New technologies, i.e., Cyber Physical Systems and blockchain are presented in Section 2. In following sections, the effect of these technologies on Maintenance 4.0 and future of maintenance strategies is presented. 


\subsection{Sustainability of Manufacturing Organizations}

Sustainable production consists of systems of production that integrate concerns for the long-term viability of the environment, worker health and safety, the community, and the economic life of a particular firm (Quinn et al., 1998). Sustainability of a manufacturing organization is measured in terms of economic, environmental and social sustainability. Some of the literature refers it as the triple bottom lines (Jovane et al., 2008). US Department of Commerce (International Trade Administration, 2007) define a sustainable manufacturing as "the creation of manufactured products that use processes that minimize negative environmental impacts, conserve energy and natural resources, are safe for employees, communities, and consumers and are economically sound". (Garetti and Taisch, 2012) defined sustainable manufacturing as the ability to use natural resources in manufacturing intelligently in order to fulfil economic, environment and social aspects and thus, preserves the environment and improve the quality of life.

Legislations in different forms have made it mandatory for manufacturing firms to consider sustainability more seriously. This is more pertinent in case of large firms that are under stricter scrutiny by the Governmental agencies. Many larger companies have implemented corporate social responsibility (CSR) programs (Fallon, 2015) for the purpose of improving, and publicizing their efforts for sustainability. These CSR and Sustainability programs help the company progress on different sustainability dimensions. Companies have started to realize the economic and strategic advantages of being sustainable. While many larger companies already have initiated some kind of CSR/CS reporting, small- and medium-sized enterprises (SMEs) usually do not possess the resources necessary to focus explicitly on sustainability, and they are also not capable of running comprehensive CSR/CS programs by a separate CSR/CS function (Winroth et al., 2016).

\subsection{OEE for Improving Sustainability}

Although, sustainability refers to the three dimensions of economics, environment and society, most of the times, it is focusses on the economic sustainability. This is especially true for small and medium firms where the financial bottom line assumes bigger importance. For example, (Pham and Thomas, 2012) presented four measures of performance, i.e. OEE, manufacturing lead time from the point of enquiry, on-time delivery, and gross value added. These are all economic sustainability measures. 
OEE as a measure of equipment effectiveness has an impact on the sustainability of the manufacturing firm. The impact of OEE is substantial in case of economic and environmental sustainability. The losses that affect the OEE are numerous, but the machine / equipment failure is the major reason. This failure also has a domino effect on the production losses like tooling, start up, etc. It is easy to see the related economic losses, but the negative impacts on ecologic sustainability cannot be neglected (Ylipaa et al., 2017). In fact, studies have shown that 30 per cent of the energy consumption in industry is wasted on machines in repair, idle, and stand-by states (Skoogh et al., 2011). According to (Yusuf et al., 2013), a reduction in energy consumption will lead to a reduction in manufacturing cost. High energy consumption impacts both the economic and environmental dimensions of the sustainability. Low OEE indicates that the utilization of current production resources is low, which in turn leads to insufficient productivity and resource efficiency. These facts are problematic for current production in terms of economic and ecologic sustainability. (Bracho, 2000) highlighted that it is crucial for manufacturers to prevent overuse of resources, which happens due to low OEE.

The role of OEE should be understood as being a measure that must be considered beyond mere monitoring and controlling (Dal et al., 2000). (Garza-Reyes et al., 2010) highlighted the importance of OEE by presenting that it prevents the sub-optimization of individual machines or production lines, provides a systematic approach for defining performance targets, takes into account process improvement initiatives, and incorporates practical management tools and techniques to achieve a balanced view of process availability, performance and quality. Similarly, (Bamber et al., 2003) remark that OEE is often used as a driver for improving the performance of a business by concentrating on quality, productivity and machine utilization issues and hence aimed at reducing non-valued adding activities often inherent in manufacturing processes. All these impacts of OEE bring about a positive change in improving the economic and environmental sustainability of the manufacturing firms.

\subsection{Essential Aspects of the CBM Strategy}

A preventive maintenance strategy, which is preferred whenever it can be applied, is the Condition Based Maintenance (CBM). The objective of CBM is to provide machines service when it is necessary (Holmberg et al., 2010; Wetzer et al., 2000). The decisions are based on the technical condition of the equipment as well as the different costs involved. 
The defects and gradual degradation processes affect the technical condition. If the degradation is revocable and economical, the condition may be repaired, but if this is not the case the replacement of parts is to be considered. The technical conditions to be taken into consideration are the factors that assure the continued trouble-free operation of the machine and its components. The financial factors are the costs of investments, operation, condition assessment and maintenance, but also the failure costs (direct costs due to primary and secondary damages, repair and loss of revenues, and indirect costs by claims or even loss of market share).

In various large sized industries, the cost of maintenance can be as much as $40 \%$ of the effective budget (Dunn, 1998; Tsang, 2002). In addition, poor quality costs are approximately $9-16 \%$ of the business turnover (Sörqvist, 1998). Also, it is known that most of the large enterprises lose approximately $2 \%$ to $16 \%$ of the annual turnover due to unplanned stoppages of their equipment (Eti et al., 2006). Moreover, the costs of poor maintenance are discussed in (Salonen and Deleryd, 2011). In conclusion, improvement of the maintenance efficiency is a potential strategic alternative for making financial savings and should be a key management issue to consider.

Therefore, the understanding of maintenance and its related factors are essential to consider. Figure 1 illustrates the production process. Its primary output is the desired product, but at the same time the production process creates demand for maintenance., which is a secondary input to production in the form of production capacity. Thus, the link should be performed efficiently, and decision-makers should be informed in time about deviation from predetermined values to be able to keep production going.

CBM uses condition monitoring to gather data for maintenance decisionmaking. For a successful CBM along with the diagnostic and test data, maintenance and other relevant data should also be used. However, there are difficulties in the application of CBM, such as the data gathered are generally

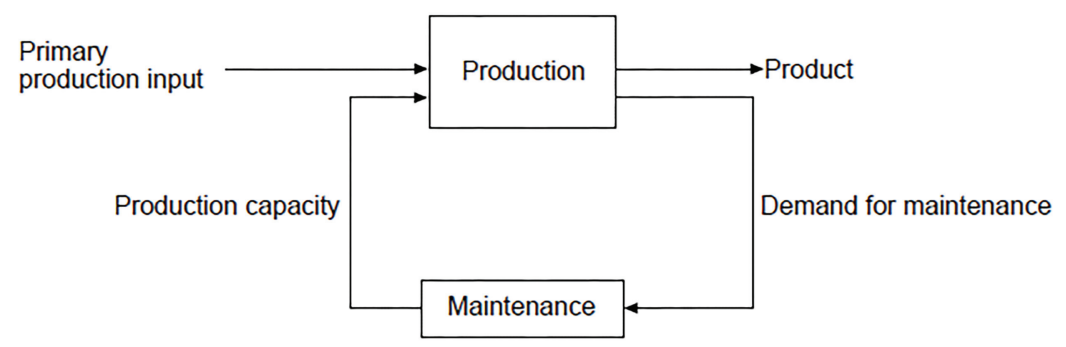

Figure 1 Production and maintenance relationship (Ben-Daya and Duffuaa, 1995). 
huge in amount, they may need to be gathered from different assets dispersed over a large geographical area, they may need to be integrated to provide any useful data and information. Also, with time, the need may be felt for data acquisition from additional sources and its integration with the rest for more meaningful interpretation and finally availability of the expert for converting data into useful information for maintenance.

To overcome these problems, people have taken option to information and communication technologies for different purposes and processes in maintenance such as decision making among others (Campos, 2009; Campos, 2016). Lately, the blockchain technology has emerged into the area to support the above mentioned technological aspects, like data/information transfer, integration, etc., which in its turn has the potential to diminish even further the costs that are related to maintenance. The blockchain technology and its place in maintenance are discussed in later sections.

\section{Enabling Technologies}

This section lays the groundwork for the envisioned approach to efficient maintenance. In particular, two technologies are considered to be the enablers for novel maintenance strategies and practices, Cyber Physical Systems and the blockchain.

\subsection{Cyber Physical Systems (CPS)}

Advances in computation and communication technologies are impacting on every aspect of people's life, and how people work is not an exception (Francis and Grootings, 2018). There is an emerging interaction between the cyber world, where the main inhabitants are data, and the physical world, inhabited by physical objects. Cyber Physical Systems act as a bridge between the two worlds, in one direction by providing data collection from the physical world to the cyber world, and actuating on physical reality based on the result of computation activities to get back to the physical reality.

From a computational point of view, a work activity is a complex of data collection, processing, and consumption. Novel techniques in industrial settings are in fact focusing on the data themselves, as an advancement over traditional model-based approaches (Krenek et al., 2016). The core of databased techniques is to take full advantage of the huge amounts of available process data, and intend to provide efficient alternative solutions for different industries, with a limited need for the modeling and configuration of the systems. 
Even though the utilization of electronics in the industry is not new, CPS allow for the integration of advanced analytics into manufacturing, products and services. In the particular use case of maintenance, a number of techniques are applied on the data, comprising smart algorithms with selfaware, self-predict, and self-configure (Lee and Bagheri, 2015). In this sense, the application of CPS to maintenance is a way to facilitate all data collection activities that empower the application of intelligent techniques, for example to profile the behavior of machinery over different conditions and look for outliers and thus predict machine's malfunctions.

CPS are thus the gateway for the data to get from the physical environment into the cyber world, and the other direction the data can take (computation results used in actuators) has usually lower importance in maintenance use cases.

Being an area that pertains inherently to applied research, most research work in the area of maintenance is driven by use cases. As an example, and quite important for this paper, in (Lee et al., 2015) the authors considered the use case of maintenance, and provided strategies and architectures to facilitate the systematic integration of CPS with machinery, and the enabling of big data analytics to look for patterns of degradations and inefficiencies in the machines. A more focused view on software is in (Algabroun et al., 2017), where the authors proposed a maintenance framework leveraging principles from self-adaptation, and focused on the maintenance of a bearing in an electrical motor.

A few works take a step back and instead of applying a synthetic approach (building a system), take on the analytic approach. For example (Albano, Ferreira et al., 2018) analyzed and categorized the types of CPS and sensors that are used in different real-life scenarios related to the maintenance use case. One of the results is that, when the machinery under analysis get more complex and expensive, the CPS and sensors get more customized, specialized on the use case at hand, and more integrated with the machine parts.

From a communication viewpoint, the application of CPS depends on the capability to transport the data between the factories where the CPS are deployed, and the cloud. In fact, modern maintenance activities are asking for the support of complex distributed systems (He and $\mathrm{Xu}, 2014)$ that collect, preprocess and transport data from the shop floor to the cloud, then use advanced techniques to distil data into information, and then get back to the shop floor to implement actions based on the data. Several advances on computation and communication technologies are the enablers to extend and adapt to the industrial context several concepts and strategies already 
applied to the personal and home environments, and on the Internet of Things (IoT) to enclose the user in a "always on, always connected" environment (Kumar et al., 2011). This gave rose to the Industrial Internet of Things (IIoT) vision, where Machine-to-Machine (M2M) communication allows in-factory machinery and remote computers to interact, to empower the technicians and managers of a company with ways to monitor and control the machines and the shop floor in general (Xu et al., 2014).

Finally, a few works address the benefits of CPS as actuators, and thus how CPS can ease common chores (Leitão et al., 2016), and in that context provided an overview of research and development challenges that must be solved to raise the Technology Readiness Level of CPS and its acceptance in the industry.

\subsection{Blockchain Technology}

The blockchain technology is a dispersed database of different records, or an archive of all transactions or digital events (Crosby et al., 2016). The importance of the blockchain technology has increased since the idea was coined in 2008 (Yli-Huumo et al., 2016). The reason why it is so popular lies in its characteristics that provide security, privacy and integrity, and also because there is no need to involve a third party that controls the transactions. For instance, the blockchain technology utilises public key cryptography whereby each agent is assigned a private key, which is kept secret like a password (Pilkington, 2016). In addition, the blockchain technology has the potential to be implemented into many fields because of its characteristics (Zheng et al., 2016). Bitcoin is the most well-known application based on blockchain, however blockchain can be applied to diverse applications far beyond cryptocurrencies.

Application of blockchain technology in industrial use cases has been focused in two main areas: the supply chain management (Abeyratne and Monfared, 2016), and the smart grid (Mengelkamp et al., 2018). These efforts aim at harvesting the appealing characteristics of the technology behind bitcoin (Hamida et al., 2017) and apply them in other application areas, and in particular want to capitalize on responsiveness (just in time production needs), traceability (ability to track position in supply chain), accountability (prevent poor quality control), and security (intellectual property and other digital asset protection).

In the supply chain that feeds any modern industry, the number of actors is high and leads to information coordination challenges. As a title of example 
(Debabrata and Albert, 2018), the factories that manufacture a product are just one of the cause for high information complexity, the others being the customer demands, the outsourcing and globalization effects, and any malicious actor that raises the need for high information confidentiality, authentication, and integrity. The application of the blockchain technology in the management of the interactions between the actors in a supply chain scenario (see Figure 2) can provide enhanced security, trust through transparency and traceability, with the enhanced flexibility enjoyed by means of decentralized data management (Abeyratne and Monfared, 2016).

The application of blockchain to the smart grid industry is instrumental in increasing the integration of renewable energy sources in the energy system (Mengelkamp et al., 2018), since renewable energy sources are inherently volatile. The distributed ledger can be used in an efficient manner to allow an energy prosumer to trade-in its energy, exchange it with the grid in exchange for incentives (e.g.: discounts on future energy consumption). Moreover, it is possible to use distributed ledgers to allow prosumers in a neighbourhood to trade in their local community the energy produced from different kinds of renewable energy sources (Basden and Cottrell, 2017). This latter use case has been implemented for example in Brooklyn, where the prosumers pay a fixed amount to the energy grid to use the energy distribution system, and are allowed to trade excess energy with the grid operator as a whole, using blockchain to account for contribution from each prosumer.

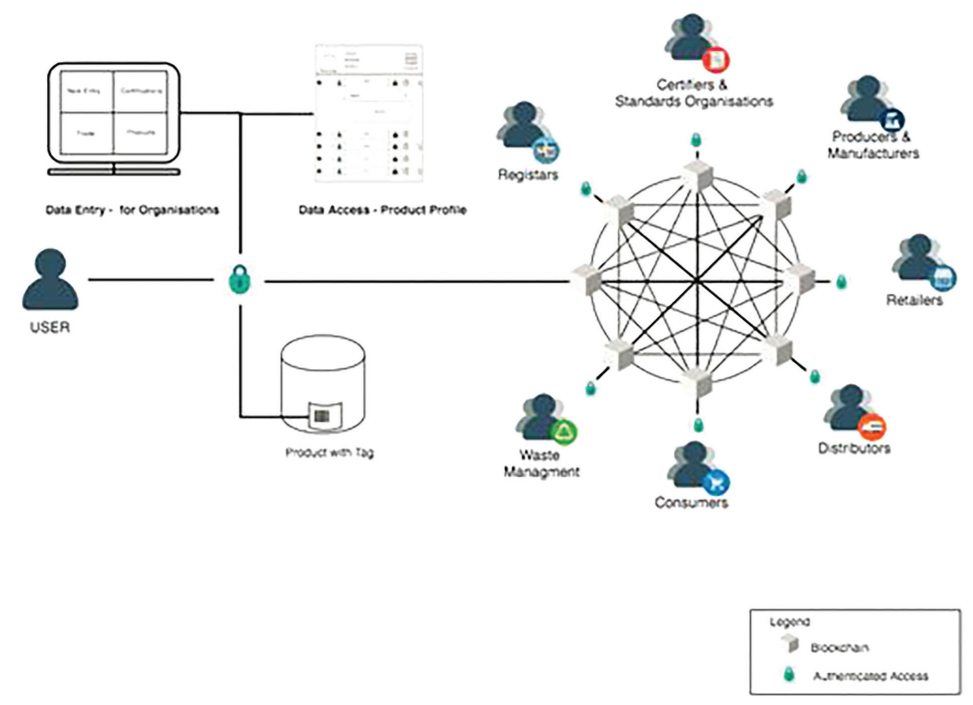

Figure 2 Blockchain in the supply chain (Abeyratne and Monfared, 2016). 
The blockchain technology contains some technical challenges and limitations that might slow down its acceptance in the future (Swan, 2015). These are, for instance, throughput, latency and usability. Throughput means that the current network in, for example, bitcoin is maximised to seven transactions per second while other similar transactions networks, such as Twitter and VISA, manage 5000 respective 2000 transactions per second. When it comes to latency, bitcoin handle each transaction in 10 seconds to be able to keep the security at an acceptable level. Usability has to do with the difficulty to use the API of, for instance, bitcoin. There is, therefore, a need to develop more user-friendly APIs for the blockchain, which might be similar to the REST APIs.

In addition to the above mentioned, there are still some issues concerning the technology that might impede its successful implementation in other areas, if not considered, such as scalability problem and privacy leakage as well as wasted resources. The scalability problems that exist may be explained by the experience of the Bitcoin, which are based on the block size having a limitation of $1 \mathrm{MB}$, and a block is mined approximately 10 minutes, which results in a network that is restricted to a speed of seven transactions per second. In the case of larger block, it would result in larger storage space and slower transmission in the network. Consequently, the compromise concerning the block size and security has become an issue to consider when the blockchain technology is intended to be implanted. In addition, the private leakage is also possible to occur even when its use only allows transactions with their public and private key (Biryukov et al., 2014). Moreover, the user's IP address is also an aspect connected with the private leakage, since it is possible to track its physical IP address. Additionally, when it comes to the wasted resources, then the algorithms such as proof of work (PoW) or proof of stake, used in the blockchain technology, are experiencing serious issues because they waste too much electricity energy, i.e. the PoW protocol is heavily energy intensive (Zheng et al., 2016; www.cryptocurrencyhub.io). Also, as the network gauges and more miners move in the mining process to handle the demand for validating transactions, the mining process becomes tougher and as a result the energy used to support the protocol increases. Consequently, because of the enormous costs of the use of PoW algorithms, there are efforts to develop alternative solutions.

A new consensus algorithm called Proof of Stake (PoS) has been suggested, which is an energy alternative to PoW (Kiayias et al., 2017). No miners exist under the PoS model. A number of PoS users stake a certain amount of a PoS currency in the blockchain's core wallet, and become the validators 
(or forgers), who are in charge of validating transactions. The new blocks are chosen deterministically, based on which participants staked more coins. The PoS offers no one-shot block rewards, and instead grant transaction fees to the validators, limiting the competition to find new blocks. Since PoS algorithms needs only need enough energy to power a blockchain's core software, the cost of running a PoS blockchain network is extremely low.

Consequently, the blockchain applications for the area of interest should be developed taking into consideration issues that existent technology might have to be able to bypass them in the developing and implementing process. Before the technology becomes more mature and has gone through some standardization processes, there is a need to find ad-hoc solutions, such as the PoS, in an effort to try to optimize the solutions of the blockchain technology that takes into consideration the energy efficacy without waste.

The blockchain technology has certain deficiencies in its scientific consistency, since it is at its nascent stage (Pilkington, 2016). However, many domains have started to understand its potential, and bitcoin is only one example of a blockchain solution. Therefore, blockchain applications have the potential to be implemented in many industries to solve different issues (Hwang et al., 2017; Chitchyan and Murkin, 2018; Larios-Hernández, 2017; Dorri et al., 2017).

It is believed that the process of adoption of the blockchain technology will be gradual and balanced (Iansiti and Lakhani, 2017). However, it will take time before it can be introduced as a substitute for already existent technologies since it is important that it first gains an overall acceptance, which is expected to result in its increased acceptance and its further successful implementation with all what it concerns. Therefore, for the blockchain to become accepted as a standard technology, it is important that there is a shared understanding by different users and business in general in connection with the workings and impact of blockchain technology (de Kruijff and Weigand, 2017).

\section{Maintenance 4.0}

Maintenance activities are changing, as many other activities in the industry, by leveraging the support of CPS, and the data they collect, which is processed by means of big data techniques to support decisions in maintenance. In fact the current industrial revolution, called Industry 4.0, is focused on the data, and on how they can be used to enhance industrial activities, from the management of the supply chain, to augment the control of industrial processes, to maintenance. In this latter context, the term Maintenance 4.0 
assumed the meaning of leveraging data for a stronger understanding of the condition of an asset, such as a manufacturing machine. This is strongly related to advanced maintenance concepts such as Condition Based Maintenance (maintenance is performed when the condition of an asset requires it) and Predictive Maintenance (data is used to predict at which point in the future and which kinds of maintenance activities will be required), and how they can be supported by Maintenance 4.0's use of data.

The full benefit of the above described technologies is taken into use to support Maintenance 4.0. One of the emphasis is on the possibility of monitoring the machinery independent where in the world it is located, by means of CPS that have internetworking capabilities such as IIoT. As described earlier, a follow up of this is the possibility for manufacturing companies to provide services for the machinery they have produced at competitive price level assuming the need for maintenance can be reliably defined. Although the term Maintenance 4.0 might suggest that all of this is a straightforward application of techniques matured in the context of Industry 4.0, it might not be the case.

CPS provide the means to have the sensors and necessary processing power installed locally. There are a number service providers who can offer the needed connection capabilities and there are also numerous platform providers that enable the collection and processing of data in the cloud. The biggest challenge in practice is not related to the measurement of the data and access to it, but the meaningful and efficient use of the data. Since the amount of data that can be collected this way is enormous, the diagnosis of the developing failures will have to be automatic otherwise the solutions are not on sound financial basis. The automation of diagnosis can be very challenging assuming that there is now previous experience of that. Technically the final goal in this process is to be able to define the remaining $\mathrm{n}$ useful life of the components automatically so that maintenance actions can be carried out at optimal time.

Many pilots have appeared in the last few years, to both showcase different concepts in Maintenance 4.0, and to work on the Technological Readiness Level of each concept. For example, Figure 3 represents the architecture of a pilot built by the project MANTIS (Ferreira et al., 2017), which defined a three-tier distributed system where CPS (on the left) collect the data, edge computing (in the middle) is used to pre-process them and prepare them for the transport to the cloud, and the cloud applies machine learning techniques to enable Condition Based Maintenance, and provides advanced visualization mechanisms, with message-based middleware connecting the three tiers. 


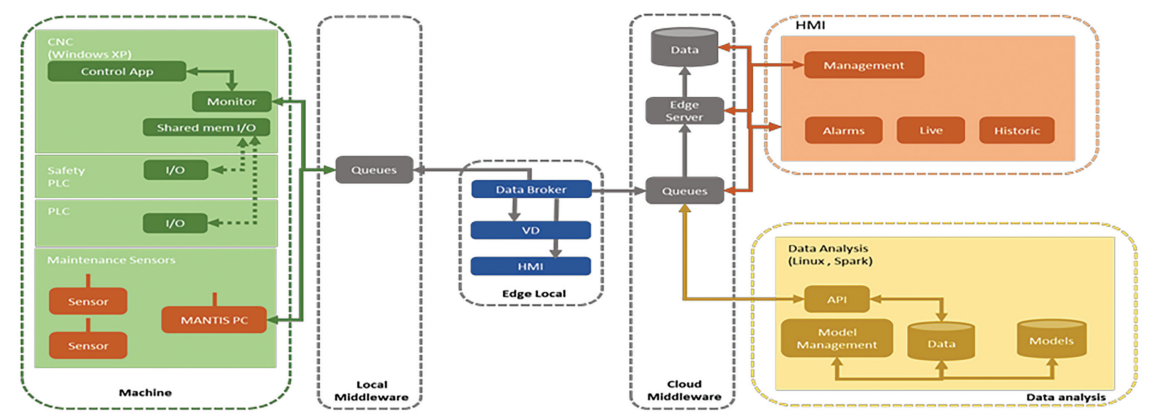

Figure 3 Reference Architecture of the MANTIS project (Ferreira et al., 2017).

Anyway, current advances are meant to be integrated in this vision to expand it and to cover novel use cases. For example, distributed ledgers can allow for non-centralized and secure management of collected data, and to support distributed decision making between different software agents in the cloud, edge computing, and human domain experts.

\section{Future of Maintenance Strategy}

From maintenance strategy point of view the technologies described in the previous chapters will totally change the situation. The data that will become available will enable the genuine introduction of CBM with the capability to predict the remaining useful life of components of machinery although it is important to remember that a lot of work will be needed in understanding the wear of the monitored components. In addition to the introduction of CBM it will be possible to tune that strategy i.e. it will be possible to try out different strategies and risk levels with components using simulation. It is fair to assume that this kind of studies can take place fully automatically so that changes in the way the CMMS system handles maintenance work orders can take place totally without human intervention. Figure 4 presents a diagrammatic representation of the future maintenance architecture. IoT will enable the machine sensors to pass the information directly to the Central Server. The central server processes the machine health data and generate documents like job card, inventory status, and other reports. These will be used by the maintenance teams to carry out the requisite maintenance tasks. All these transactions will be based on the blockchain, thereby leading to an online distributed record of the interactions which are very beneficial for a green environment. All these facets form part of a viable Cyber Physical System for asset maintenance. 


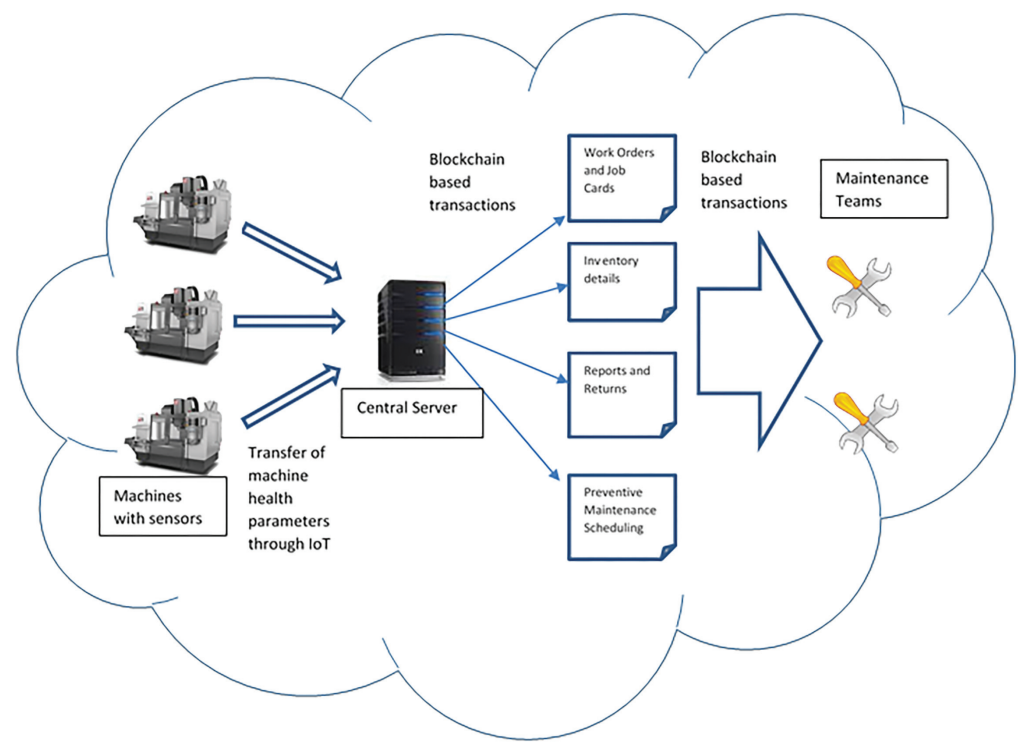

Figure 4 Advancing maintenance with CPS and blockchain.

Related to the above the OEE value can be followed on-line all the time which will lead to dramatic growth of awareness of how well the machines are working. Nobody will in the future accept OEE values that are below 50\%. Instead the goal will be set to high values in the order of more than $90 \%$. This can then lead to dramatic reduction of the load the industry provides the nature globally. It should be remembered that in all cases when the OEE has been measured with some accuracy it has always been a surprise how low it is in these cases which can be considered to represent the front line of industry what are interested in this kind of monitoring. Consequently it is only possible to try to guess how low the real values are in majority of companies in the whole world. Could it be that the average of OEE in all industrial sectors in the whole world is below $50 \%$ today which could mean that load the industry creates to the nature today might be reduced to half with the same level of produced outcomes.

Naturally all of the above cannot come true overnight and without investments but reduction of the price of sensors and processing power and technologies like the blockchain and cloud technology will make it possible for companies that could not even dream about these technologies in the past. Consequently, the authors believe that the wheel has started to rotate and will rotate with increasing speed and that no other industrial improvement like 
e.g. improvements in energy production can have a similar size of influence to the stop the global warming.

Computerized maintenance management systems (CMMS) have found widespread usage in the modern maintenance organizations. However, the companies are still stuck with a large number of paper-based transactions that are causing delays and mistakes. These setbacks are resulting in dilution of the advantages that CMMS can otherwise accrue. Modern automated maintenance systems still work in hybrid mode where critical activities follow the paper transactions. Maintenance managers use CMMS to print out work-orders and distribute the paper copies to the maintenance team. The maintenance teams complete the repair/maintenance work and fill the details in the work-orders. Maintenance managers receives the completed work-orders and makes the relevant entries into the CMMS and closes work-orders. These data entry actions can consume as much as two hours a day for the maintenance manager, with some additional time consumed in filling the paper work-order by the maintenance teams. This amounts to nearly 500 manhours per year. This nonvalue-added time can be better spent delivering real value to the organization by optimizing maintenance schedules, analyzing past work orders for trends and streamlining the maintenance function to deliver better reliability at the same or at a lower cost.

Manual entry of data to the CMMS can also cause a lot of mistakes. Incorrect entries made by the maintenance crew or the maintenance manager can result in wrong analysis of the past trends. These mistakes can either be of omission or commission. Deliberate entry of wrong data can jeopardize maintenance programmes for critical systems.

From the energy consumption standpoint, previous work have proved that the mean energy communication cost over the internet can be estimated between 0.05 and 0.09 Joules/Byte (Gupta and Singh, 2003). Thus, if a number of stakeholders must be contacted, for example to cross-verify each transaction that enables the supply chain behind maintenance, the energy expenditure sums up. From the time consumption standpoint, it is possible that some of the stakeholders act as bottlenecks, for example on front of system downtimes, leading to long delay times.

Blockchain based transactions can obviate these problems. The maintenance block chain is initiated by the maintenance manager. The maintenance crew continues to add blocks to the chain as and when a new repair activity is performed on the asset. As is the property of the blockchain, these transactions are non-deletable and can only be appended. This will create a permanent record of activities with a time-stamp on each of the transaction. 


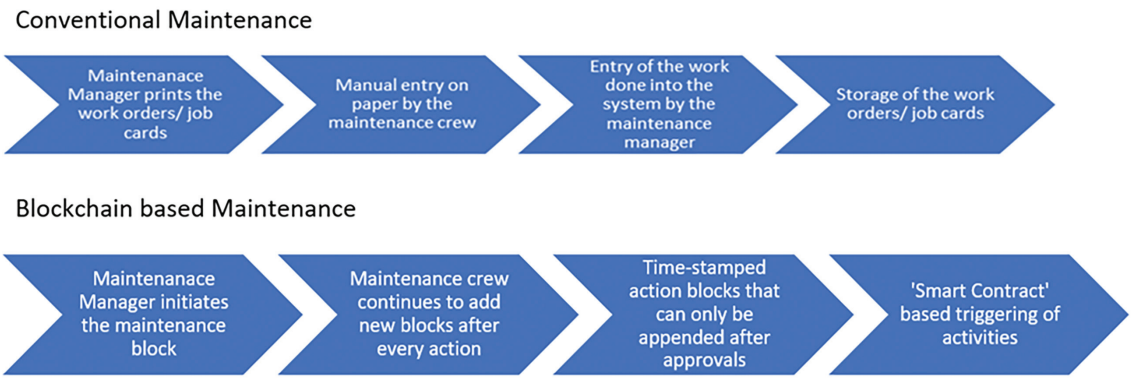

Figure 5 Conventional maintenance strategy versus blockchain based maintenance.

Such a system will ensure that non-value-added time of doing paperwork is eliminated; thereby resulting in a reduction in operating expenses at each stage of the maintenance chain. 'Smart Contracts' will ensure that subsequent activities like release of spare part, ordering of fresh inventory, etc. is triggered automatically on completion of certain milestones without having someone to actually do it manually. System rules can be engrained that can dispose the transactions off without manual intervention.

Implementation of such a system will not be without its fair share of problems. Any enterprise comprises of humans who are resistant to change. Legacy ways of working have become so entrenched within daily operations, that the organizations lack the ability to look beyond existing systems and processes. In addition to this, decision-makers are often trapped by the responsibilities of daily operations. Despite these issues, the top management should focus on the strategic advantage of implementing a blockchain based system that can reap benefits in the longer run.

\section{Conclusion}

The blockchain technology provides several benefits especially in the form of improved OEE, and it is, therefore, important to understand how to introduce it smoothly into the domain of interest. It is important to learn from the experience of the previous emergent technologies and how they have been introduced and accepted successfully. It has been shown that it is usually done by the standardisation of the technologies, which leads to their rapid acceptance as well as an increased use in the industry respectively. However, the standardization of the blockchain technology is somewhat important to avoid it becoming a technological hype. 
The resulting vision is an extension of the current understanding of the potential of application of Cyber Physical Systems to collect data for Maintenance 4.0, since the distributed ledger allows for a decentralized trustable data management (see Figure 5), and in this sense allows to reduce communication activities and third-party actions during data collection and processing. Thus, it allows for savings in terms of energy used in the communication, and time needed for the convergence of the data and mutual assurance on its validity.

\section{Acknowledgement}

This work was partially supported by National Funds through FCT/MEC (Portuguese Foundation for Science and Technology), Finnish Funding Agency for Innovation Tekes, and co-financed by ERDF (European Regional Development Fund) under the PT2020 Partnership, within the CISTER Research Unit (CEC/04234); also by FCT/MEC and the EU ECSEL JU under the H2020 Framework Programme, within project ECSEL/0004/2014, JU grant nr. 662189 (MANTIS).

\section{References}

[1] Abeyratne, S. A., and Monfared, R. P. (2016). Blockchain ready manufacturing supply chain using distributed ledger. International Journal of Research in Engineering and Technologies, 5(9), 1-10.

[2] Albano, M., Ferreira, L. L., di Orio, G., Malo, P., Webers, G., Jantunen, E., Gabilondo, I., Viguera, M., Papa, G. and Novak, F. (2018). Sensors: the Enablers for Proactive Maintenance in the Real World. In 5th International Conference on Control, Decision and Information Technologies (CoDIT'18), 10-13, Thessaloniki, Greece.

[3] Albano, M., Jantunen, E., Sharma, P. Campos, J., Baglee, D. (2018). Blockchain Technology Helps Maintenance to Stop Climate Change. The Maintenance, Performance, Measurement and Management Conference 2018 (MPMM 2018), 10-17, 21-23, Coimbra, Portugal.

[4] Algabroun, H., Iftikhar, M. U., Al-Najjar, B., and Weyns, D. (2017). Maintenance 4.0 Framework Using Self-Adaptive Software Architecture. In Proceedings of 2nd International Conference on Maintenance Engineering (IncoME-II 2017), 299-309, The University of Manchester, UK. 
[5] Bamber, C., Castka, P., Sharp, J. and Motara, Y. (2003). Cross-functional team working for overall equipment effectiveness. Journal of Quality in Maintenance Engineering, Vol. 9(3), 223-239.

[6] Basden, J. and Cottrell, M. (2017). How utilities are using blockchain to modernize the grid. Harvard Business Review.

[7] Ben-Daya, M. and Duffuaa, S. O. (1995). Maintenance and quality: the missing link. Journal of quality in maintenance engineering, 1(1), 20-26.

[8] Biryukov, A., Khovratovich, D. and Pustogarov, I. (2014). Deanonymisation of clients in Bitcoin P2P network. In Proceedings of the 2014. ACM SIGSAC Conference on Computer and Communications Security, 15-29. ACM.

[9] Blanchard, B. S. (1997). An enhanced approach for implementing total productive maintenance in the manufacturing environment. Journal of Quality in Maintenance Engineering, Vol. 3(2), 69-80.

[10] Bracho, F. (2000). The future of oil and energy: consequences for oil producing countries. Foresight, Vol. 2(4), 379-390.

[11] Campos, J. (2009). Development in the application of ICT in condition monitoring and maintenance. Computers in Industry, 60(1), 1-20.

[12] Campos, J. (2016). Managing the information systems in the industrial domain. Cogent Business \& Management, 3(1), 1180967.

[13] Chitchyan, R. and Murkin, J. (2018). Review of Blockchain Technology and its Expectations: Case of the Energy Sector. arXiv preprint arXiv:1803.03567.

[14] Crosby, M., Pattanayak, P., Verma, S. and Kalyanaraman, V. (2016). Blockchain technology: Beyond bitcoin. Applied Innovation, 2, 6-10.

[15] Dal, B., Tugwell, P. and Greatbanks, R. (2000). Overall equipment effectiveness as a measure of operational improvement - a practical analysis. International Journal of Operations \& Production Management, Vol. 20(12), 2000, 1488-1502.

[16] de Kruijff, J. and Weigand, H. (2017). Understanding the blockchain using enterprise ontology. In International Conference on Advanced Information Systems Engineering (29-43), Springer, Cham.

[17] Debabrata, G., and Albert, T. (2018). A Framework for Implementing Blockchain Technologies to Improve Supply Chain Performance. SCALE Working Paper Series.

[18] Dorri, A., Kanhere, S. S., Jurdak, R. and Gauravaram, P. (2017). Blockchain for IoT security and privacy: The case study of a smart home. In 2017 IEEE International Conference on Pervasive Computing and Communications Workshops (PerCom Workshops), (618-623). IEEE. 
[19] Dunn, S. (1998). Reinventing the maintenance process: towards zero downtime. In Queensland Maintenance Conference Proceedings, Queensland, Australia.

[20] Edward, H., and Hartmann, P. E. (1992). Successfully Installing TPM in a Non-Japanese Plant. TPM Press, Pittsburgh, PA.

[21] Ericsson, J. (1997). Disruption Analysis- An important tool in Lean production. Department of Production and Materials Engineering, Lund University, Lund, Sweden.

[22] Eti, M. C., Ogaji, S. O. T. and Probert, S. D. (2006). Reducing the cost of preventive maintenance $(P M)$ through adopting a proactive reliabilityfocused culture. Applied energy, 83(11), 1235-1248.

[23] Fallon, N. (2015). 22 Great examples of socially responsible businesses. Business News Daily.

[24] Ferreira, L. L., Albano, M., Silva, J., Martinho, D., Marreiros, G., di Orio, G., Malo, P., and Ferreira, H. (2017). A pilot for proactive maintenance in industry 4.0. In IEEE 13th International Workshop on Factory Communication Systems (WFCS), 1-9.

[25] Francis, A., and Grootings, P. (2018). New Technologies and Work: Capitalist and socialist perspectives. Vol. 15. Routledge.

[26] Garetti, M. and Taisch, M. (2012). Sustainable manufacturing: trends and research challenges. Production Planning \& Control, Vol. 23 (2-3), 83-104.

[27] Garza-Reyes, J. A. (2015). From measuring overall equipment effectiveness (OEE) to overall resource effectiveness (ORE). Journal of Quality in Maintenance Engineering, Vol. 21(4), 506-527.

[28] Garza-Reyes, J. A., Eldridge, S., Barber, K. D. and Soriano-Meier, H. (2010). Overall equipment effectiveness (OEE) and process capability (PC) measures - a relationship analysis. International Journal of Quality and Reliability Management, Vol. 27 (1), 48-62.

[29] Gupta, M. and Singh, S. (2003). Greening of the Internet. In Proceedings of the 2003 conference on Applications, technologies, architectures, and protocols for computer communications. 19-26. ACM.

[30] Hamida, E. B., Brousmiche, K. L., Levard, H. and Thea, E. (2017). Blockchain for Enterprise: Overview, Opportunities and Challenges. In The Thirteenth International Conference on Wireless and Mobile Communications (ICWMC 2017).

[31] $\mathrm{He}, \mathrm{W}$. and $\mathrm{Xu}, \mathrm{L} . \mathrm{D}$. (2014). Integration of distributed enterprise applications: A survey. IEEE Transactions on Industrial Informatics 10.1 (2014): 35-42. 
[32] Holmberg, K., Adgar, A., Arnaiz, A., Jantunen, E., Mascolo, J. and Mekid, S. (2010). E-maintenance. London: Springer. ISBN 978-1-84996205-6.10.1007/978-1-84996-205-6

[33] Hwang, J., Choi, M. I., Lee, T., Jeon, S., Kim, S., Park, S. and Park, S. (2017). Energy Prosumer Business Model Using Blockchain System to Ensure Transparency and Safety. Energy Procedia, 141, 194-198.

[34] Iansiti, M. and Lakhani, K. R. (2017). The truth about blockchain. Harvard Business Review, 95(1), 118-127.

[35] Ingemansson, A. (2004). On reduction of production disturbances in manufacturing systems based on discrete-event simulation. $\mathrm{PhD}$ thesis, Department of Mechanical Engineering, Lund University, Lund.

[36] International Trade Administration (2007). How does commerce define sustainable manufacturing? US Department of Commerce. available at: www.trade.gov/competitiveness/sustainablemanufacturing/how_doc _defines_SM.asp

[37] Jeong, K. -Y. and Phillips, D. T. (2001). Operational efficiency and effectiveness measurement. International Journal of Operations \& Production Management, Vol. 21(11), 1404-16.

[38] Jovane, F., Yoshikawa, H., Alting, L., Boër, D. R., Westkämper, E., Williams, D., Tseng, M., Seliger, G. and Paci, A. M. (2008). The incoming global technological and industrial revolution towards competitive sustainable manufacturing. CIRP Annals, Vol. 57(2), 641-659.

[39] Kiayias, A., Russell, A., David, B., and Oliynykov, R. (2017). Ouroboros: A provably secure proof-of-stake blockchain protocol. In Annual International Cryptology Conference, 357-388. Springer, Cham.

[40] Kotze, D. (1993). Consistency, accuracy leads to maximum OEE benefits. TPM Newsletter, Vol. 4(2).

[41] Krenek, J., Kuca, K., Blazek, P., Krejcar, O. and Jun, D. (2016). Application of artificial neural networks in condition based predictive maintenance. In Recent Developments in Intelligent Information and Database Systems (pp. 75-86). Springer, Cham.

[42] Kumar, S., Kadow, B. and Lamkin, M. (2011). Challenges with the introduction of radio-frequency identification systems into a manufacturer's supply chain - A pilot study. Enterprise Inform. Syst., vol. 5(2), 235-253.

[43] Larios-Hernández, G. J. (2017). Blockchain entrepreneurship opportunity in the practices of the unbanked. Business Horizons, 60(6), 865-874.

[44] Lee, J. and Bagheri, B. (2015). Cyber-Physical Systems in Future Maintenance. 9th WCEAM Research Papers. Springer, Cham, 299-305. 
[45] Lee, J., Ardakani, H. D., Yang, S. and Bagheri, B. (2015). Industrial Big Data Analytics and Cyber-physical Systems for Future Maintenance \& Service Innovation. Procedia CIRP, Volume 38, 3-7.

[46] Leitão, P., Colombo, A. W. and Karnouskos. S. (2016). Industrial automation based on cyber-physical systems technologies: Prototype implementations and challenges. Computers in Industry 81: 11-25.

[47] Mengelkamp, E., Notheisen, B., Beer, C., Dauer, D. and Weinhardt, C. (2018). A blockchain-based smart grid: towards sustainable local energy markets. Computer Science-Research and Development, 33(1-2), 207-214.

[48] Nakajima, S. (1998). An Introduction to TPM. Productivity Press, Cambridge, MA.

[49] Parida, A., Kumar, U., Galar, D. and Stenström, C. (2014). Performance measurement and management for maintenance: a literature review. Journal of Quality in Maintenance Engineering, Vol. 21(1), 2-33.

[50] Pham, D. T. and Thomas, A. J. (2012). Fit manufacturing: a framework for sustainability. Journal of Manufacturing Technology Management, Vol. 23(1), 103-123.

[51] Pilkington, M. (2016). Blockchain technology: principles and applications. In: Olleros, F. X., Zhegu, M. (eds.) Research Handbook on Digital Transformations. Edward Elgar, Northampton.

[52] Quinn, M. M., Kriebel, D., Geiser, K. and Moure-Eraso, R. (1998). Sustainable production: a proposed strategy for the work environment. American Journal of Industrial Medicine, 34(4), 1998, 297-304.

[53] Robinson, C. J. and Ginder, A. P. (1995). Implementing TPM: The North American Experience. Productivity, Portland, OR.

[54] Salonen, A. and Deleryd, M. (2011). Cost of poor maintenance: A concept for maintenance performance improvement. Journal of Quality in Maintenance Engineering, 17(1), 63-73.

[55] Skoogh, A., Johansson, B. and Hansson, L. (2011). Data requirements and representation for simulation of energy consumption in production systems. Proceedings of the 44th CIRP, Conference on Manufacturing Systems, Madison, WI, 1-3. 
[56] Sörqvist, L. (1998). Poor quality costing (Doctoral dissertation, KTH).

[57] Swan, M. (2015). Blockchain: Blueprint for a new economy. O'Reilly Media, Inc.

[58] The, Y. and Johnston, S. (2015). Benefits of Overall Equipment Effectiveness (OEE) techniques in metal mining environments. Schneider Electric White Paper, 1-11.

[59] Tsang, A. H. (2002). Strategic dimensions of maintenance management. Journal of Quality in Maintenance Engineering, 8(1), 7-39.

[60] Wetzer, J. M., Cliteur, G. J., Rutgers, W. R., and Verhaart, H. F. A. (2000). Diagnostic and condition assessment-techniques for condition based maintenance. Conference on Electrical Insulation and Dielectric Phenomena, Vol. 1. 47-51.

[61] Winroth, M., Almström, P. and Andersson, C. (2016). Sustainable production indicators at factory level. Journal of Manufacturing Technology Management, Vol. 27 (6), 842-873.

[62] Xu, L. D., He, W. and Li, S. (2014). Internet of things in industries: A survey. IEEE Transactions on industrial informatics, 10(4), 2233-2243.

[63] Yli-Huumo, J., Ko, D., Choi, S., Park, S. and Smolander, K. (2016). Where is current research on blockchain technology? - a systematic review. PloS one, 11(10), e0163477.

[64] Ylipaa, T., Skoogh, A., Bokrantz, J. and Gopalakrishnan, M. (2017). Identification of maintenance improvement potential using OEE assessment. International Journal of Productivity and Performance Management, 66(1), 126-143.

[65] Yusuf, Y. Y., Gunasekaran, A., Musa, A., El-Berishy, N. M., Abubakar, T. and Ambursa, H. M. (2013). The UK oil and gas supply chains: an empirical analysis of adoption of sustainable measures and performance outcomes. International Journal of Production Economics, 146(2), 501514.

[66] Zheng, Z., Xie, S., Dai, H. N. and Wang, H. (2016). Blockchain challenges and opportunities: A survey. Work Pap. 


\section{Biographies}

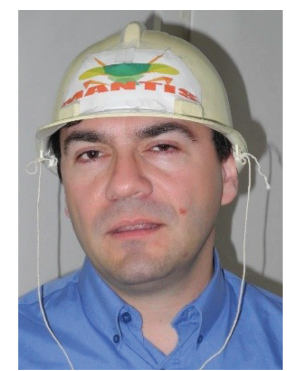

Michele Albano is a Research Associate in the CISTER Research Unit of the Polytechnic of Porto, Portugal, and Adjunct Professor at Polytechnic Institute of Cavado e Ave, Portugal. His research focuses on Cyber Physical Systems and Communication Middleware, in particular for the application areas of industrial maintenance, smart grids, and vehicular networks.

Michele received his degrees from the University of Pisa, Italy, and he was researcher at Universidad de Malaga, Spain, at University of New York at Stony Brook, and at Instituto de Telecomunicações in Aveiro, Portugal. He has been involved in more than 10 international research projects, and he is currently active in the context of European projects Productive 4.0 and MANTIS, and in the latter he is Work Package leader for WP3 "Smart sensing and data acquisition technologies" and for WP8 "Dissemination of knowledge and exploitation". His works have been published in more than 70 international conferences and journals, and he is the editor of the book The MANTIS book: Cyber Physical System Based Proactive Maintenance. Since 2015, Michele is the Editor in Chief for the Journal of Green Engineering, River Publishers. 


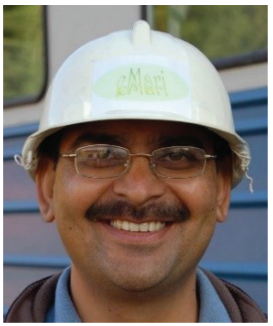

Pankaj Sharma is a Research Fellow at the Centre for Next Generation Logistics at the National University of Singapore. He is currently working on creating digital twins for the Warehouses for optimizing the resource utilization. His $\mathrm{PhD}$ thesis is in the field of Spare Parts forecasting and Selective Maintenance Optimization. He is an MTech from the same Institute in Industrial Engineering. His research interests are in Maintenance Strategy Development, Supply Chain Management and Logistics. He had participated in project e-Mari at VTT Finland as an inter-university exchange student.

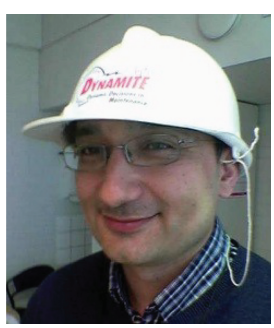

Jaime Campos is an associate professor at the Department of Informatics, Linnaeus University, Sweden. His main research interests include the Information and Communication Technologies in the industrial domain, mainly in asset management. 


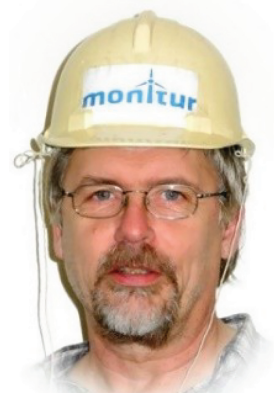

Erkki Jantunen is principal scientist at VTT Technical Research Centre of Finland. He has been a member of the editorial board and acted as a reviewer of a number of scientific journals. He has been project manager of many research projects. He is the author and co-author of several books and more than 150 research papers in the field of condition monitoring, diagnosis and prognosis and e-maintenance. He has a position as a visiting professor at the University of Sunderland. 